\section{Tumorinfiltrierende Lymphozyten als Prognoseparameter bei Brustkrebs}

\begin{abstract}
Viele Biomarker sind prädiktiv oder prognostisch als wichtige Größen etabliert. Ob eine verstärkte Tumorinfiltration durch Lymphozyten auf eine bessere Prognose hinweist, war die Frage einer multinationalen Studie.
\end{abstract}

— orscher um Sherene Loi untersuchten 2.009 Tumorproben, die im Rahmen der Studie BIG 02-98 entnommen worden waren. In dieser Studie war in mehreren Ansätzen die Addition von Docetaxel zu einer Anthrazyklin-basierten adjuvanten Chemotherapie bei nodalpositiven Mammakarzinomen gestestet worden. Zwei unabhängige Pathologen analysierten mikroskopisch das Tumormaterial in der konventionellen Hämatoxilin-Eosin-Färbung und bestimmten den Grad der Lymphozyteninfiltration in das Epithel der invasiven Tumorzellnester sowie in das Stromagewebe, das die Tumorzellen umgab.
Bei der Analyse aller Tumoren ergab sich zunächst keine signifikante Assoziation zwischen dem Grad der Lymphozyteninfiltration und der Prognose der Patientinnen.

Bei Östrogen- und HER-2-Rezeptornegativen Karzinomen jedoch reduzierte jede Zunahme der intratumoralen Infiltration um $10 \%$ das Rezidivrisiko um $17 \%$ ( $\mathrm{p}=0,1$ als Trend) sowie das Mortalitätsrisiko um $27 \%(\mathrm{p}=0,035)$. Analog war eine um $10 \%$ vermehrte intrastromale Lymphozyteninfiltration mit einer Abnahme des Rezidivrisikos um 15\% ( $\mathrm{p}=$ $0,025)$ und des Mortalitätsrisiko um $17 \%$ $(\mathrm{p}=0,023)$ assoziiert.
Bei HER-2-positiven Tumoren zeigte die Analyse einen signifikanten Zusammenhang zwischen der $10 \%$-igen Steigerung der Lymphozyteninfiltration im Stroma und dem rezidivfreien bzw. dem Gesamtüberleben zugunsten einer Chemotherapie ohne Docetaxel (Signifikanzniveau für Interaktion: 0,042 bzw. 0,018).

Fazit: In der vorliegenden Studie erwies sich für verschiedene Tumor-Subtypen die Infiltration von Tumorzellnestern und Stroma als günstiger prognostischer Marker. Ob bzw. wann dieser neue Ansatz zukünftig auch therapeutische Entscheidungen beeinflussen wird, bleibt jedoch abzuwarten. Andreas Fischer

Loi $S$ et al. Prognostic and predictive value of tumor-infiltrating lymphocytes in a phase III randomized adjuvant breast cancer trial in nodepositive breast cancer comparing the addition of docetaxel to doxorubicin with doxorubicinbased chemotherapy: BIG 02-98. J Clin Oncol. 2013;31(7):860-7.

Fazit: Die Inzidenz mittelschwerer bis schwerer später Toxizitäten einer 3DCRT bleibt bei längerer Nachbeobachtungszeit erheblich, besonders subkutane Fibrosierungen und Fettnekrosen. Darunter leidet das kosmetische Ergebnis dosisabhängig auf das bestrahlte Volumen bezogen. Unterschiede in den Fraktionsintervallen spielen wahrscheinlich keine Rolle. Friederike Klein bei $31 \%$ der Frauen auf, vom Grad 3/4 bei $7,5 \%$. Nur bei fünf Patientinnen (6\%) besserten sich die Fibrosen mit zunehmendem zeitlichem Abstand von der Bestrahlung. $11 \%$ der Frauen entwickelten Fettnekrosen. Das kosmetische Ergebnis wurde von den Untersuchern nach der Harvard-Skala bei $29 \%$ der Frauen als exzellent, bei $52 \%$ als gut, aber bei $10 \%$ als mäßig und bei $9 \%$ als schlecht bewertet. Das Risiko einer subkutanen Fibrose war mit dem relativen Brustvolumen, das $5 \%, 20 \%, 50 \%, 80 \%$ oder $100 \%$ (V5V100) der geplanten Strahlendosis erhalten hatte, assoziiert, ein mäßiges oder schlechtes kosmetisches Ergebnis mit V50 bis V100. Das Intervall zwischen den Fraktionen (im Median 6,9 Stunden, Minimum 6,2 Stunden) war für die erfassten Parameter der späten Toxizität nicht bedeutsam.
Leonard KL et al. The effect of dose-volume parameters and interfraction interval on cosmetic outcome and toxicity after 3-dimensional conformal accelerated partial breast irradiation. Int J Radiat Oncol Biol Phys. 2013;85(3):623-9.

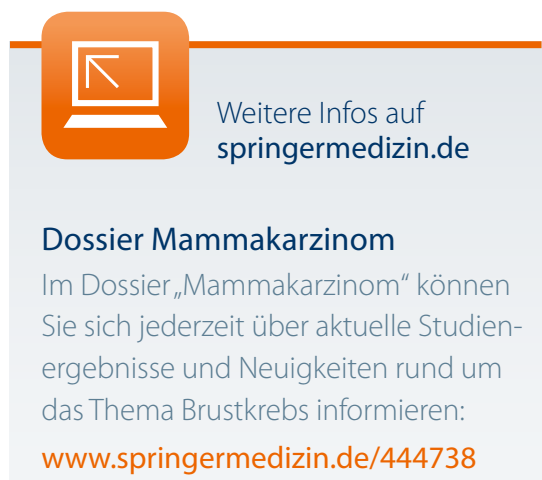

